\title{
Simulation for Improvement Plan of the Fluidized Bed Structure in the Bulk Cement Tanker Based on FLUENT
}

\author{
Pei Weichi ,Han Ju, Li Yaogang \\ Hebei United University, \\ Tangshan, China \\ pwc@heuu.edu.cn,Hanju3721@163.com,jxlyg@heuu.edu.cn
}

\begin{abstract}
Structural parameters of the fluidized bed is the most important components to influence the performance index of the bulk cement tankers. The paper is focused on some types of bulk cement tank truck, proposed to a research programs to improve the fluidization quality of the fluidized bed that substitute Y-type fluidization board by rectangle fluidization board. Created the fluidization models of this improvement plan in the CFD (Computational Fluid Dynamics) software FLUENT, then simulated for improved fluidization process, and given the analysis for fluidization quality. The simulation results show that the rectangle fluidization board are able to improve the fluidization quality of the cement in tanker. The improvement of their structure in the original basis of bulk materials vehicles can improve their serviceability and work efficiency.
\end{abstract}

Keywords-Fluidized bed, Bulk cement tanker, Simulation, FLUENT

\section{INTRODUCTION}

The theory of bulk cement tanker operation is the air compressor on vehicle bringing compressed air through the medium of fluid bed, causing cement powder fluidization in tank, and then fluidized cement powder to destination through deliver conduit. The key of improving the bulk cement tanker performance is the quality of fluidization, increasing transport efficiency and decreasing remainder rate[1]. To optimize the structural parameters of the fluidized bed can improve the fluidization quality effectively.

\section{Distinguish MeAns OF FLUIDIZATION QUALiTY}

The so called fluidization is compressed air through the medium of fluid bed, making cement powder on fluidized bed separate from each other and suspended in the air, it has characteristics of general fluid. There are several ways to evaluate the quality of fluidization [2]:

Froude Number used to Determine the Particulate Fluidization and Aggregative Fluidization.

Particulate fluidization:

$$
F r_{m f}=\frac{u_{m f}^{2}}{g d_{p}}<0.13
$$

Aggregative fluidization:

$$
F r_{m f}=\frac{u_{m f}^{2}}{g d_{p}}>1.3
$$

Gas-solid Particulate Fluidization Criterion.

$$
F r_{m f} \operatorname{Re}_{m f}\left(\frac{\rho_{p}-\rho_{f}}{\rho_{f}}\right)\left(\frac{H_{m f}}{D}\right)<100
$$

According to Eq.1, Eq.2 and Eq.3, input related data can get the Froude number of bulk cement tanker $F r_{m f}=0.022, R e_{m f}=0.012, H_{m f}$ is $1900.8 \mathrm{~mm}, D$ is $2466 \mathrm{~mm}$, then the empirical formula of the bulk cement tanker is 0.41 .

So we can find that in the current operating conditions, particulate fluidization occurs in tanker.

It is difficult to directly ascertain the velocity of gasphase in 2-phase system as per upwards analyses. Therefore it needs the result of numerical calculation to estimate the type of fluidization and the quality of fluidization.

\section{STUDY OF IMPROVING FLUIDIZATION QUALITY}

Through the analysis of simulation result about fluidized process of fluidized bed in current tanker, several improvement programs are proposed:

1. substitute Y-type fluidization board by rectangle fluidization board.

It will be make a blast of efflux on the rectangle grid plate, and it will be make 2 effluxes on the Y-type of plate when air through the fluidization board. The two effluxes will gradually mix into a blast of efflux when the distance between two plates of Y-type fluidization board is nearby. At this moment, the height of two effluxes after mixed almost close the bed height. It can take a large stirring action to the bed and make the fluidization quality better. But with the height increase of the fluidization board, the distance of two plate of Y-type fluidization board will be increased too. So the two effluxes will not mixed but increased respectively. Since the height of the bed has been relatively low, the height of two effluxes almost throughout the whole bed, it will make the short circuit of the air, and worsened the fluidization quality. So it needs modify the structure of fluidization board to improve the fluidization quality under all things being equal.

2. Substitute rectangle fluidization board by curve fluidization board.

The efflux can be destroyed, making the compressed air through which the grid plate can change the trend of upward to lateral flow, the air which through the plates and cement 
powder will be fully mixed and the dead bands near the plates will be decreased, when using the curve board.

Simulation of this program in FLUENT indicates that the type of board can eliminate the dead band of splitter board effectively. Furthermore, there is no channel in middle of fluidized bed after the air through the board, it can avoid the short circuit of the discharge port connected. At same time this type of board caused the fluidization quality decreases at the middle zone of fluidized bed.

3. Make the fluidized more fully with a larger gasinlet velocity.

Gasinlet velocity has great influence on the quality of fluidization. It is important to choose the gasinlet velocity for fluidization quality after the bed structure, bed height and performance parameters of air and powder are defined. Either in rectangular or curve section, expansion of the fluidized bed changed little when the gasinlet velocity is $0.13 \mathrm{~m} / \mathrm{s}$. Consider about the power consumption of air compressor, gasinlet velocity can be increased to $0.2 \mathrm{~m} / \mathrm{s}$.

Simulation results in FLUENT show that fluidized process is prompt and adequate in section when gasinlet velocity is $0.2 \mathrm{~m} / \mathrm{s}$. momentum of air and cement have great changes in section and dead band are decreased within the fluidized bed. But still have some unique characteristics like asymmetric structure of fluidization, middle efflux and so on.

4. Change the angle of the side splitter board to improve the quality of fluidization.

It will make dead band and remnant increase when the angle of side splitter board is smaller than the static angle of internal friction. If the angle is too big then the effective volume of the tank will reduce, causing the economiy reduce too.

Compared to $42^{\circ}$ of the original design of the quality of fluidization, choose $40^{\circ}$ and $44^{\circ}$ cases in simulation. The results show that the fluidization quality of $40^{\circ}$ is higher than the other two cases.

\section{Simulation FOR the Fluidization EFFECT}

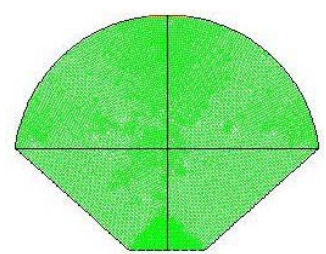

Figure 1. Reference line position on section

Take the above-mentioned measurement namely substitute Y-type fluidization board by rectangle fluidization board. Simulation in FLUENT. In order to improve computational efficiency, it needs to establish 2-dimension feature section as the model of the fluidized process of the fluidized bed. Figure. 1 shows the cement volume fraction of the reference position on the specific location line within the section.

After analysis, the fluidized process has similar trend in the 2-dimensional model and 3-dimensional model. 3- dimensional model of the fluid degree is greater than 2dimensional at same computation time, and poor fluidization quality zone near the fluidization board can use cement powder velocity of 2-D model to estimate the same area in 3-D model. For these reasons, the cement fluidized process model in tanker can be created in FLUENT, the boundary conditions shows in Tablele 1. Calculated using separate solvers, transient solution and iterative time step is $0.001 \mathrm{~s}$, calculated time is $30 \mathrm{~s}$; take the largest section of the fluidized bed depth as the feature section [3]. Simulation result shows in Figure. 2 4.

Contrast the cross-section of top of Y-type fluidization board, volume fraction changes of cement at different moment in Figure. 2 shows that the fluidization quality of rectangular board is better than the Y-type one. The reason is that efflux not run through whole the bed although the height of efflux through the rectangular is also larger. So it can avoid the worsened of bed fluidization quality caused by short circuit of air.

The air and cement velocity vector distribution within section in Figure. 3 show that speed values in rectangular fluidization board is larger than the values in Y-type board. This shows that height momentum exchange are in progressed by cement and air inner bed.

The distribute of volume fraction of cement on reference line in section in Figure. 4 shows, although some areas still remain not fully fluidization, but the fluidization areas of whole bed on top of rectangular board far greater than the Y-type board one.

Volume change of cement at different time shows that efflux focuses on middle of fluidization board from beginning to $10 \mathrm{~s}$ and gradually excursion to both sides form $10 \mathrm{~s}$ to $30 \mathrm{~s}$. These phenomena in favor of avoid air short circuit between discharge port and fluidization board at dumping course. It also shows that bias of the right and left side of the fluidization board along cross section profile are reduced over time, while the more uniform in inflation time. Effectively overcome the strong right bias when the splitter board's angle is $42^{\circ}$, improve the quality of fluidization. Finally, cement is fluidized in most of the section, and the fluidization degrees are more fully than low gasinlet velocity.

The air and cement velocity vector distribution within section show that air horizontal flow through the fluidized bed.

the average volume fraction of cement iner bed is 0.42 and average volume fraction of cement near the side flitter board is 0.35 , optimization effect is better than individual measures in above-mentioned.

Other improvements plans as the mentioned above, so need not repeat the description.

\section{V. . SUMMARIES}

The improvement plan which substitute Y-type fluidization board by rectangle fluidization board, adopt $40^{\circ}$ side splitter board, with curve fluidization board substitute rectangle board as well as use larger gasinlet velocity are able to improve the fluidization quality of the 
cement in tanker. This plan can be used to all bulk materials vehicles giving priority to bulk cement tanker, to improve their structure in the original basis of bulk materials vehicles can improve their serviceability and work efficiency.

\section{REFERENCES}

[1] PEI Wei-chi; LI Yao-gang; HAN Ju; LI Ji-shun. Study and Simulation for Optimization Plan of the Fluidization Quality of the
Bulk Cement Tanker Device. Applied Mechanics \& Materials [J], 2010, PP:1249-1253

[2] PEI Wei-chi; LI Yao-gang. The Simulation for the Fluidization Process of the Bulk Cement Tanker Based on Fluent. Machine Tool \& Hydraulics[J],2008,PP:153-156.

[3] YU Zhou-sheng Application of Pneumatic Conveying[M],1989

[4] HAN Zhan-zhong Application and Simulation Examples of Fluid Engineering in FLUENT [M],2004

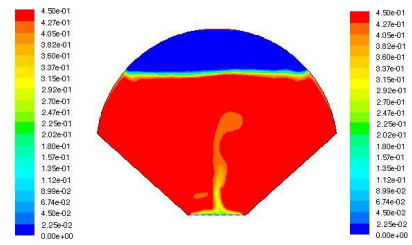

(a) $5 \mathrm{~s}$

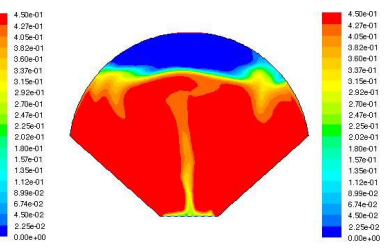

(b) $10 \mathrm{~s}$

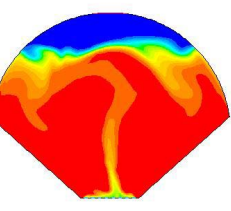

(c) $15 \mathrm{~s}$

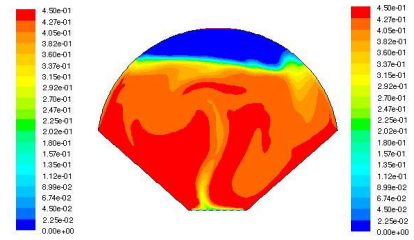

(a) $10 \mathrm{~s}$

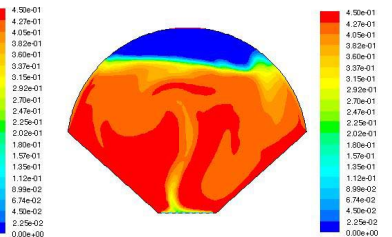

(b) $20 \mathrm{~s}$

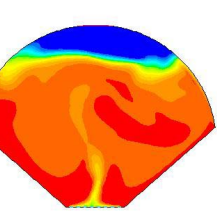

(c) $30 \mathrm{~s}$

Figure 2. The volume fraction of cement in section at different computing time
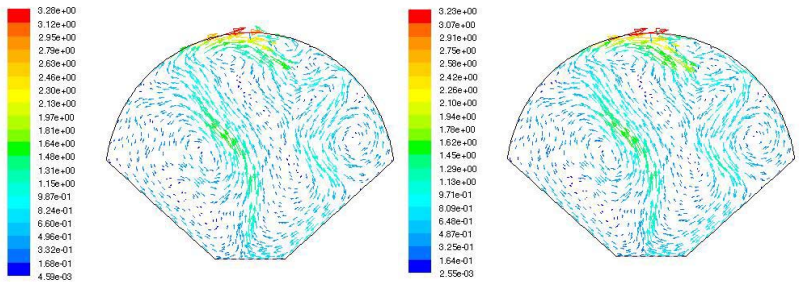

(a) air

(b) cement

Figure 3 . Vector of velocity in section at $30 \mathrm{~s}$

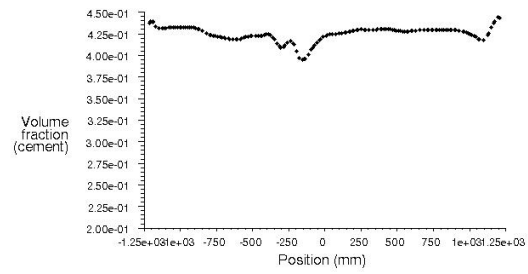

(1) horizontal line

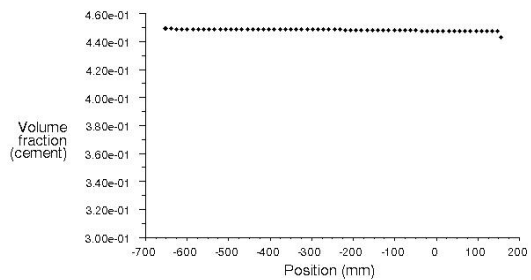

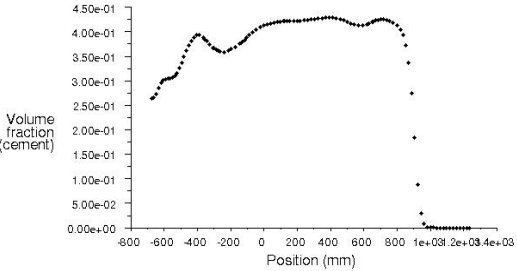

(2) vertical line

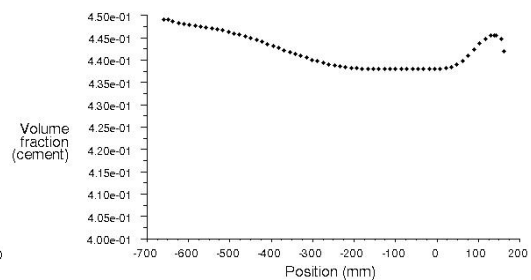

Figure 4. Volume fraction of cement on reference line in section 
TABLE I. BASIC DATA OF CALCULATE NUMERICAL

\begin{tabular}{cccccccc}
\hline $\begin{array}{c}\text { Cement } \\
\text { Density } \\
\left(\mathbf{k g} / \mathbf{m}^{3}\right)\end{array}$ & $\begin{array}{c}\text { Powder } \\
\text { Diameter } \\
(\mathbf{m m})\end{array}$ & $\begin{array}{c}\text { Powder } \\
\text { Viscosity } \\
(\mathbf{P a . s})\end{array}$ & $\begin{array}{c}\text { Air } \\
\text { Density } \\
\left(\mathbf{k g} / \mathbf{m}^{3}\right)\end{array}$ & $\begin{array}{c}\text { Air } \\
\text { Viscosity } \\
(\mathbf{P a . s})\end{array}$ & $\begin{array}{c}\text { Gasinlet } \\
\text { Velocity } \\
(\mathbf{m} / \mathbf{s})\end{array}$ & $\begin{array}{c}\text { Outlet } \\
\text { Pressure } \\
(\mathbf{P a})\end{array}$ & $\begin{array}{c}\text { Operating } \\
\text { Pressure } \\
(\mathbf{P a})\end{array}$ \\
\hline 3100 & 0.05 & 0.001 & 1.51845 & 1.51845 & 0.13 & 0 & 1.51845 \\
\hline
\end{tabular}

